\title{
A STATISTICAL ANALYSIS ON RECOVERY CASES OF COVID19 IN INDIA
}

\author{
Jaydip Datta * \\ *Indian Institute of Chemical Engineers ( CRC ) , J.U campus , 700032 , INDIA \\ ( Email : deepjoy1972@gmail.com )
}

\begin{abstract}
-
In this report the positive cases of Covid19 in India with effect from $7^{\text {th }}$ September, 2020 to $25^{\text {th }}$ October, 2020 are analysed for statistical relevance. The scattered data are used to find out a model equation correlating two variables number of recovered Covid -patient with an interval of regular seven days. The best fit regression analysis shows a significant correlation of Pearson coefficient $(r)$ with standard error $(s)$ with a probable lower mortality rate. Finally the limitations of this analysis is discussed herewith.
\end{abstract}

Keywords - Covid19, Best fit regression, Hyperbolic fit, Recovery rate , Reproducibity of research INTRODUCTION :

The important statistical parameters used to explain an epidemiology like Covid19 better Known as mortality rate (MR ), case fatality rate (CFR ), Infection fatality rate(IFR)[ $1,2,4$ ]. The mathematical model like Susceptibity - Infectivity - Recovery ( SIR ) [ 3 ] used mostly to quantify the different epidemiological stages. The stages are namely viral inoculation, spreading of viral infection and finally recovery from Covid19. The statistical models from best fit data analysis also used to explain the above epidemiological parameters ( MR , CFR , IFR ) are nonlinear regression modelling like hyperbolic regression, nth order polynomial regression, MMF regression, logistic or sigmoid regression . In this article the final stage of SIR modelling, Recovery stage[ 3 ] is analysed by best fit regression .

\section{METHODS AND DISCUSSION [ 6 ]:}

1. Data Availability - a set of data (Figure - I) of number of days with seven days interval vs number of recovered cases with effect from $7^{\text {th }}$ September $-25^{\text {th }}$ October, 2020 .

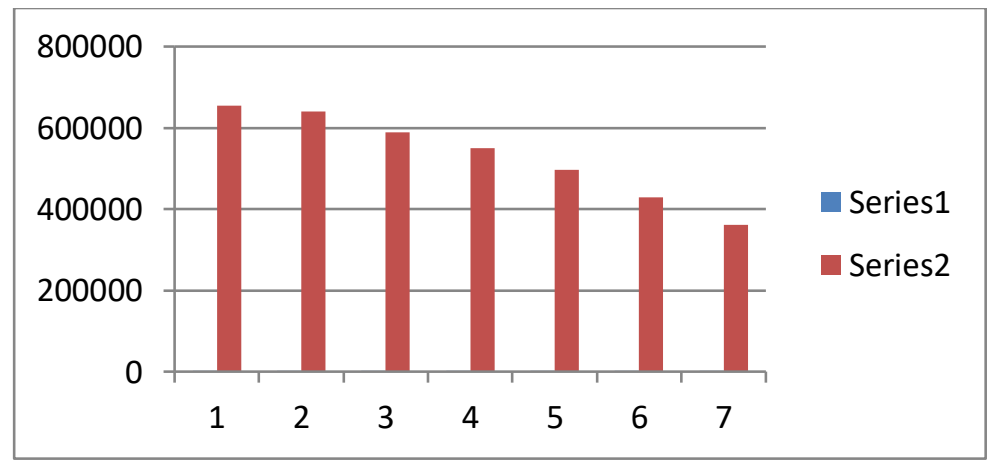

Figure - I 


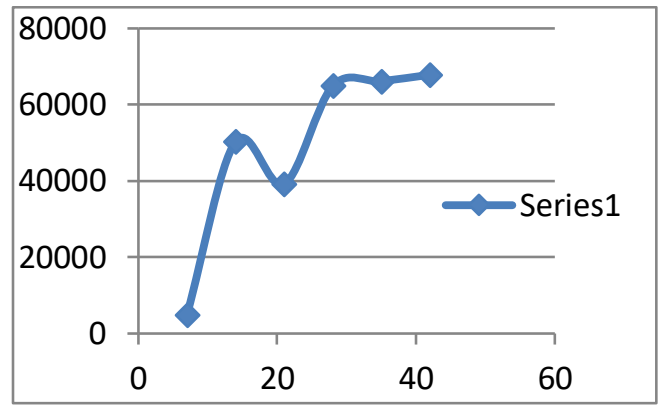

Figure - I : Excell plot of scattered data ( 5 ) of number of days with seven days interval ( $x$-axis ) vs number of positive Covid-cases ( $y$-axis )

Data Informatics Tool: Standard Statistical Software Curve Expert v.1.4.

RESULTS AND DISCUSSIONS [ 7,8,9,10]: The results may be considered as output of the standard software analysed by a best fit regression methods to calculate the most important statistical parameters like model equation, Pearson coefficient $(r)$, standard error ( $s)$, chi-sq relevance, covariance matrix and residual table.

\section{The output of software after execution of dataset}

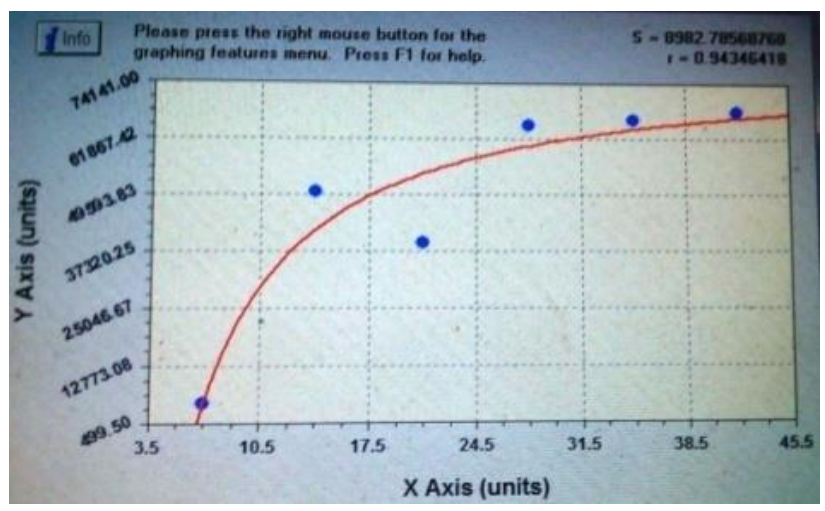

( Figure -II )

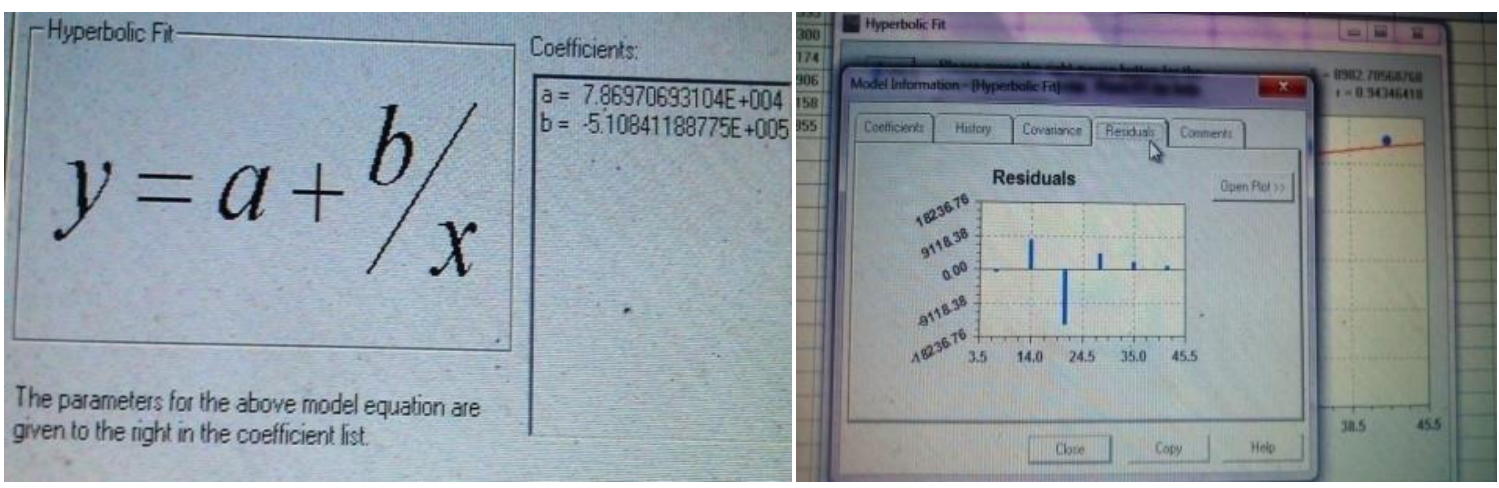



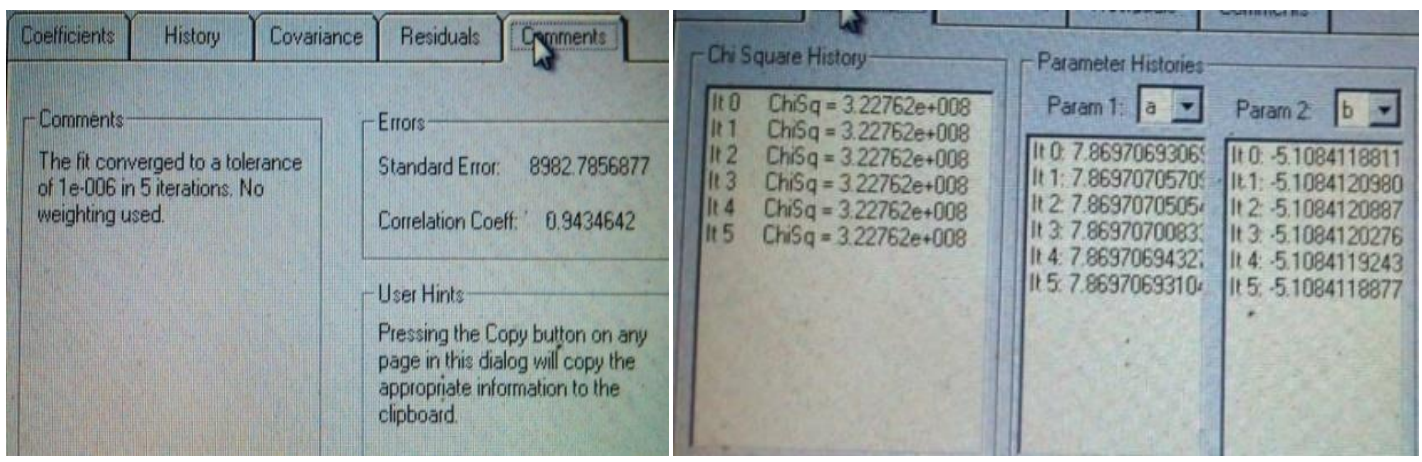

Figure -II : $t$-axis - time in number of days, $n$-axis - number of recovered cases in each seven days

interval. The model rate equation [ time in days,$t=f($ number of recovered cases,$n)$ can be executed as hyperbolic best fit( 2 ) as follows :

$\mathrm{t}=7.8697 * 10^{\wedge} 4-\left(5.1084^{*} 10^{\wedge} 5 / \mathrm{n}\right)$ ( equation 1.0 )

[ Where $a=7.8697 * 10^{\wedge} 4, b=5.1084^{*} 10^{\wedge} 5$ are called coefficient of hyperbolic fit ]

\section{CONCLUSION $[9,10,11]$ :}

The best fit statistical analysis of hyperbolic fit (equation 1.0) resumes a significant Pearson coefficient ( $r$ ), standard error ( $s$ ). The statistical data execution may be analysed as collection of Covid19 recovery data to fit in a standard statistical software like Curve Expert 1.4 to get the hyperbolic fit in Figure -II by calculating Chi-Sq hypothesis, covariance matrix $-2 \times 2$, Residual mean , the final result of correlation coefficient $(r)$ with a standard error, equal residuals , $r=0.9464(0<r<1)$, $S=$ standard error= 8983-1* $10^{\wedge}(-6)$ iterative convergence ]. Although the correlation signifies the relevant recovery rate with a probably low mortality rate [4]. The major limitations of this theoretical method is that lower frequency, only six numbers of dataset with irregular scattered data explains the poor biological reproducibility [ 11 ] of SARS-COV-2 mutants to predict the relationship of long term recovery of active SARS-COV-2 patients naturally very difficultly predict the mortality rate [ 4 ].

\section{REFERENCE :}

1. COVID19 - A Correlation Study of Infection Fatality Rate vs Age, Jaydip Datta, DOI: https://doi.org/10.21203/rs.3.rs-85482/v2 .

2. Growth of D614G Virus : Morgan - Morgan - Finney ( MMF ) Regression, Jaydip Datta , DOI: https://doi.org/10.21203/rs.3.rs-92776/v1.

3. Mathematical and Statistical Study on COVID19- SIR Model, Jaydip Datta, DOI: https://doi.org/10.21203/rs.3.rs-89766/v2 .

4. https://en.wikipedia.org/wiki/Mortality rate

5. Data Source -Sharpest weekly fall as cases down 16\%, toll $19 \%$ / Man hits docs over Covid death, -Oct 26, 2020,

https://health.economictimes.indiatimes.com/?utm source=Mailer\&utm medium=-- 


\section{utm_medium--\&utm_campaign=--utm_campaign--]}

6..https://towardsdatascience.com/understanding-regression-using-covid-19-dataset-detailedanalysis-be7e319e3a50.

7.https://arxiv.org/abs/2004.05684, kisor sahu , April ,2020 .

8 .https://www.medrxiv.org/content/10.1101/2020.05.29.20117069v1 - May,2020 .

9. COVID19 DRUG REVIEW DISCUSSIONS (Version Version 1.0). Zenodo, http://doi.org/10.5281/zenodo.4244641, JAYDIP DATTA , 2020.

10.https://www.academia.edu/44413464/SUPPLIMENTARY RESOURCES Health Statistics Applied To Covid19 Recovery kinetics, Jaydip Datta .

11.

https://www.researchgate.net/post/Let us Discuss something about Reproducible research techniqu e including COVID 19 ,( Jaydip Datta ) , RG , 2020. 